\title{
COMPLEXIFICATION, TWISTOR THEORY, AND HARMONIC MAPS FROM RIEMANN SURFACES
}

\author{
BY MICHAEL G. EASTWOOD
}

\begin{abstract}
Penrose's twistor theory and many other ideas of mathematical physics are based on the notion of complexification. This notion is explained and examples of its application in physics and mathematics are described. In particular, the well-known analogy between Yang-Mills fields and harmonic maps of Riemann surfaces becomes rather stronger after complexification. This strengthening is the main point of this paper.
\end{abstract}

Introduction. Throughout Penrose's development of twistor theory [25, 28], complexification is omnipresent, albeit often only implicitly. This technique is probably more familiar to physicists than mathematicians, especially its informal use in quantum field theory. It is, however, a quite precise construction which can probably find greater application in mathematics than it presently enjoys. The idea is that many structures based on real numbers become more understandable if viewed in a complex environment. Familiar examples are algebraic varieties and trigonometric or elliptic functions. In $\$ 1$ a brief exposition of the complexification of real-analytic structures is given, together with a few examples.

The main examples of this article, however, come from twistor theory and the theory of harmonic maps as discussed in $\$ \$ 2$ and 3 respectively. As regards twistor theory, the Ward correspondence for self-dual Yang-Mills fields emerges as a direct analogue of the Cauchy-Riemann equations for Riemann surfaces. The harmonic maps of $\$ 3$ will be from a Riemann surface into complex projective space. The construction of such mappings due to Din and Zakrzewski [7, 8] and, independently, Burns [4] (see also Eells and Wood [11, 12]) becomes rather clearer after complexification. This clarification and the strengthening of the well-known analogy between Yang-Mills fields and harmonic maps are two of the aims of this article. Also, this gives an illustration of the utility of complexification.

This utility is well known to those who know and in particular to Roger Penrose and Claude LeBrun (see [18-20]), to whom I am grateful for many useful conversations. I would also like to thank the Institut des Hautes Études Scientifiques for hospitality during the work described in this paper.

1. Complexification. Suppose $M$ is a real-analytic manifold. Each coordinate patch $U \subset \mathbb{R}^{n}$ can be enlarged to a neighbourhood $\mathbb{C} U \subset \mathbb{C}^{n}$. Since the coordinate changes are real analytic functions, they may be extended to these

Received by the editors January 4, 1984.

1980 Mathematics Subject Classification. Primary 53B35; Secondary 53B50.

${ }^{1}$ Research supported by an SERC Advanced Fellowship.

(C)1984 American Mathematical Society 0273-0979/84 \$1.00+\$.25 per page 
enlarged neighbourhoods (assumed sufficiently small for the relevant power series still to converge) and, as shown by Bruhat and Whitney [2], if care is taken to ensure the Hausdorff condition then the result is a complexification of $M$. More precisely, this process creates a complex manifold $\mathbb{C} M$ equipped with a conjugate holomorphic mapping ${ }^{-}: \mathbb{C} M \rightarrow \mathbb{C} M$ called complex conjugation, whose fixed point set is $M$. As a germ around $M, \mathbb{C} M$ is unique and can be called the complexification. Infinitesimal constructions on $M$ complexify as tensoring with $\mathbb{C}$ so that, for example, $\left.T \mathbb{C} M\right|_{M}=\mathbb{C} T M$, where $T$ means tangent bundle and $\mathbb{C} T M \equiv \mathbb{C} \otimes_{\mathbb{R}} T M$. Real-analytic structures on $M$ extend uniquely to the complexification by first expressing them in terms of power series transition rules. For example, a real-analytic connection on a realanalytic vector bundle will become a holomorphic connection on a holomorphic vector bundle on $\mathbb{C} M$. The original structure can always be recovered by means of a complex conjugation or reality structure. Some examples of complexification are as follows.

Suppose $M$ is a two-dimensional Riemannian manifold. Gauss considered the question of whether one could always find local coordinates $x$ and $y$ in which the metric took the form $f(x, y)\left(d x^{2}+d y^{2}\right)$ for some positive function $f$. He was motivated by the question of whether one can make a map of a piece of countryside so that angles are preserved. The affirmative answer in the smooth case, due to Korn and Lichtenstein, is not simple (see Chern [5]), but Gauss proved only the real-analytic version, in which case complexification provides an elegant and geometric proof. To motivate this proof first consider the case in which $M$ is Lorentzian, i.e. suppose $M$ has a metric of signature $(+,-)$ and ask whether it locally takes the form $f(x, y)\left(d x^{2}-d y^{2}\right)$. In this case there are at each point of $M$ two directions singled out by the metric, namely the null directions, where the corresponding tangent vectors have zero length. Locally one can make a consistent choice (indeed, globally, if $M$ is orientable) to obtain two direction fields which integrate to give two transverse foliations by curves. Using these curves as local coordinates $(u, v)$, the metric takes the form $f d u d v$, and then set $u=x+y, v=x-y$. To mimic this proof in the Riemannian case choose an orientation and define $J \in \operatorname{Aut}\left(T^{*} M\right)$ to be rotation through $\pi / 2$ in the sense of the orientation. The problem is to find local coordinates so that $J(d x)=-d y$ and $J(d y)=d x . J$ is the Hodge *-operator. For the corresponding construction in the Lorentzian case, $J^{2}=1$ and the null covectors are the \pm 1 -eigenspaces. In the Riemannian case $J^{2}=-1$ so $J$ has no real eigenvectors. If, however, $M$ and $J$ are real-analytic then $J$ extends to some neighbourhood of $M$ in its complexification $\mathbb{C} M$ where it now has genuine eigenvectors. $J$ has eigenvalues $\pm i$ since this is the case on $M$. Just as in the Lorentzian case the eigenspaces induce foliations by curves, in this case holomorphic foliations by holomorphic curves. Hence there is a holomorphic coordinate $z$ such that $J(d z)=i d z$. Restricting to $M$ and writing $z=x+i y$ gives $J(d x)=-d y$ and $J(d y)=d x$ as required.

To summarize Gauss' theorem: an oriented real-analytic manifold $M$ with conformal structure (a Riemannian metric defined only up to scale) is equivalent to a Riemann surface. In retrospect it is possible to be more explicit about 
C $M$, namely $\mathbb{C} M=M \times \bar{M}$, where $M$ denotes $\bar{M}$ but with the conjugate holomorphic structure. The real slice $M$ is embedded as the (antiholomorphic) diagonal $M \ni m \mapsto(m, \bar{m}) \in M \times \bar{M}$, and the usual decomposition of the complexified tangent bundle of $M$ according to type, $\mathbb{C} T M=T^{1,0} M \oplus T^{0,1} M$, becomes exactly the splitting of the tangent bundle to $\mathbb{C} M$ as $T \mathbb{C} M=T M \oplus$ $T \bar{M}$. This geometric interpretation of $T^{p, q} M$ gives a simple way of seeing that a vector bundle on $M$ may be given a holomorphic structure by means of a $\bar{\partial}$-operator. To be more precise, suppose $E$ is a real analytic complex vector bundle on $M$ and $D: \Gamma(E) \rightarrow \Gamma\left(E \otimes T^{0,1} M\right)$ satisfies $D(f s)=f D s+s \otimes \bar{\partial} f$ for $s \in \Gamma(E)$ and $f$ a smooth function. Supposing also that $D$ is real analytic, $E$ and $D$ extend to some neighbourhood of $M$ in $\mathbb{C} M$ where one can interpret $D$ as a connection in the $\bar{M}$ direction. Since $\bar{M}$ is one dimensional, $D$ is flat so $E$ is canonically trivialized in the $\bar{M}$ direction and hence identified with a holomorphic vector bundle on $M$ as required.

Similar arguments apply to manifolds of higher dimension as follows. An almost complex manifold is a smooth manifold $N$ together with an automorphism $J: T N \rightarrow T N$ such that $J^{2}=-1$. If $N$ is a complex manifold then one can take $J$ to be multiplication by $i$. Conversely, under suitable integrability conditions, $J$ defines a complex structure on $N$. Although the proof, in general, due to Newlander and Nirenberg [22] is difficult, the case of $N$ and $J$ real-analytic is clear after complexification (as noted by Eckmann and Fröhlicher [9]). Just as for surfaces consider the $\pm i$-eigenspaces of $J$ extended to $\mathbb{C} T N$. On $N$ this is the splitting $\mathbb{C} T N=T^{1,0} N \oplus T^{0,1} N$. The only difference in the general case is that these distributions may not be integrable in the sense of Frobenius. Thus $J$ defines a complex structure when $\left[T^{0,1} N, T^{0,1} N\right] \subset T^{0,1} N$. A consequence of the Newlander-Nirenberg theorem is that an integrable almost complex structure is real analytic, i.e. there is an analytic structure for $N$ subordinate to the given smooth structure such that $J$ is real-analytic. Malgrange has shown how to deduce this directly from the theory of nonlinear elliptic systems, so the above proof by complexification is valid for the smooth case (see Nirenberg [24]). Just as for surfaces it is clear retrospectively that if $N$ is complex then $\mathbb{C} N=N \times \bar{N}$ (the complex structure on $\bar{N}$ being prescribed by $-J)$. A holomorphic vector bundle on $N$ may be regarded as a holomorphic vector bundle on $\mathbb{C} N$ with a flat connection in the $\bar{N}$ direction. This may be recovered from data on the real slice, namely a real-analytic complex vector bundle on $N$ together with $D: \Gamma(E) \rightarrow \Gamma\left(E \otimes T^{0,1} N\right)$ satisfying

$$
D(f s)=f D s+s \otimes \bar{\partial} f \text { and } D^{2}=0,
$$

where $D: \Gamma\left(E \otimes T^{0,1} N\right) \rightarrow \Gamma\left(E \otimes T^{0,2} N\right)$ is characterized by

$$
D(s \otimes \omega)=D s \wedge \omega+s \otimes \bar{\partial} \omega
$$

for $s \in \Gamma(E)$ and $\omega \in \Gamma\left(T^{0,1} N\right)$.

The passage from smooth to real analytic is not always possible. For example, a smooth hypersurface in a complex manifold acquires the remnants 
of a complex structure, namely a CR-structure (see, for example, [29]). If such a structure is given and is real-analytic then, conversely, it is easy to show by complexification that it is realizable as such a hypersurface. This conclusion is false even locally in the smooth category (e.g. [24, 29, 20]).

In $\$ 3$ the example of complexification as applied to a Kähler manifold (specifically, complex projective space) will be needed. The extra structure present on such a complexification is as follows. The manifold itself complexifies as $\mathbb{C} N=N \times \bar{N}$. Letting $\Theta^{1,0}$ (resp. $\Theta^{0,1}$ ) denote the holomorphic tangent bundle of $N$ (resp. $\bar{N}$ ), the tangent bundle to $\mathbb{C} N$ is $\Theta^{1,0} \oplus \Theta^{0,1}$. The Hermitian metric on $N$ is best regarded as a real Riemannian metric (, ) compatible with the complex structure in that $(J X, Y)+(X, J Y)=0$. Therefore, upon complexification, $($,$) becomes a holomorphic metric and compatibility becomes$ that $\Theta^{1,0}$ and $\Theta^{0,1}$ should be isotropic. The Kähler condition on $N$ can be stated as $\nabla J=0$, where $\nabla$ is the Levi-Civita connection for $($,$) . Upon$ complexification this is the condition that the holomorphic connection $\nabla$ preserve the splitting $\Theta^{1,0} \oplus \Theta^{0,1}$, i.e. $\nabla$ is the direct sum of an affine connection on each of $\Theta^{1,0}$ and $\Theta^{0,1}$.

Other important examples of complexification are for compactified Minkowski space and the sphere $S^{4}$ together with their conformal structures. These examples are the basis of twistor theory and are discussed in $\$ 2$.

Complexification as used in mathematics occurs, for example, in Grauert's [13] proof that a real-analytic manifold may be embedded in some $\mathbb{R}^{N}$ as a closed real-analytic submanifold. The main step in this proof is in showing that the complexification can always be taken to be Stein. Complexification is also a first step in hyperfunction technology [30].

In physics, complexification is employed repeatedly in quantum field theory and especially in Hawking's [14] path-integral approach to quantum gravity. The idea is that if the time coordinate $t$ is formally replaced by imaginary time $i \times t$, then many previously ill-defined and divergent procedures become convergent and the answers can then be analytically continued back to real time. For example, the Schrödinger equation

$$
\Delta \Psi+V \Psi=i \partial \Psi / \partial t
$$

becomes the heat equation with potential,

$$
\Delta \Psi+V \Psi=\partial \Psi / \partial t
$$

which is mathematically much easier to handle. In general relativity the procedure is somewhat more vague but the idea is to replace the Lorentzian metric by a Euclidean one, which is again mathematically more convenient. In this context it is interesting to note the recent theorem of DeTurck and Kazdan [6], which states that, parallel with Malgrange's theorem on the real analyticity of integrable almost complex structures, any smooth Riemannian solution of the Einstein vacuum equations admits a complexification. Other parallels between complex structures and Einstein manifolds are discussed by Koiso [17].

2. Twistor theory. Let $V$ be a complex vector space and $\mathbb{P}(V)$ the corresponding projective space. Denote by $Q$ the nonsingular quadric in $\mathbb{P}(V)$ defined by $f(Z, Z)=0$ for $f \in \odot^{2} V^{*}$. A hyperplane tangent to $Q$ intersects in 
a singular quadric, and decreeing that this quadric defines null directions at the point of tangency equips $Q$ with a canonical holomorphic conformal structure. To be more precise, if $f$ is used to identify the normal bundle to $Q$ with $\mathcal{O}(2)$ and the Euler sequence

$$
\mathcal{O} \rightarrow \mathcal{O}(V)(1) \rightarrow \Theta
$$

is used to study the tangent bundle to $\mathbb{P}(V)$, then the tangent bundle to $Q$ may be identified as

$$
T Q=\{\Phi \in \mathcal{O}(V)(1) \text { s.t. } f(\Phi, Z)=0\} /\{\Phi=Z \phi\} .
$$

Hence, there is a symmetric form

$$
\begin{array}{ccc}
T Q \otimes T Q & \rightarrow & \mathcal{O}(2) \\
\Psi & & \Psi \\
(\Phi, \Psi) & \rightarrow & f(\Phi, \Psi),
\end{array}
$$

noting that this is independent of choice of $\Phi$ and $\Psi$. A local trivialization of $\mathcal{O}(2)$, i.e. a choice of conformal factor, gives a local holomorphic metric and hence a global conformal structure (though there is no global representative metric). It is easy to check that $Q$ of dimension $n$ is a conformal compactification of $\mathbb{C}^{n}$ with holomorphic metric $d z_{1}^{2}+\cdots+d z_{n}^{2}$.

In the special case of $n=2, Q$ is just $\mathbb{P}_{1} \times \mathbb{P}_{1}$, where $\mathbb{P}_{1}$ is the Riemann sphere. The conformal metric is null in the directions of the factors. As in $\$ 1$ the Riemann sphere may be embedded as the antiholomorphic diagonal. Thus, $Q$ provides a complexification of $S^{2}$ with its conformal structure. Another real slice of $Q$ is the torus $S^{1} \times S^{1}$ for $S^{1}$ the equator of $\mathbb{P}_{1}$. In this case the conformal metric on the slice is Lorentzian.

The example relevant to twistor theory is $n=4$, where $Q$ provides a complexification of $S^{4}$, the conformal compactification of Euclidean 4-space [1], or a complexification of the conformal compactification of Minkowski space [25]. The constructions of twistor theory $(n=4)$ often have analogies with similar constructions for $n=2$. In particular, it is interesting to view the Ward correspondence [31] in this light as follows.

Suppose more generally that $M$ is a Riemann surface with complexification $\mathbb{C} M=M \times \bar{M}$. A holomorphic 1 -form on $\mathbb{C} M$ splits according to type, $\omega=\omega^{1,0}+\omega^{0,1}$, where $* \omega^{1,0}=i \omega^{1,0}$ and $* \omega^{0,1}=-i \omega^{0,1}$. Consider the problem of finding a 1-form which is closed and self-dual, i.e.

$$
d \omega=0 \text { and } * \omega=i \omega
$$

Locally one can always find a potential for $\omega$, i.e. a holomorphic function $\phi$, unique up to the gauge freedom of an additive constant, such that $d \phi=\omega$. Then self-duality for $\omega$ becomes $\bar{\partial} \phi=0$, the Cauchy-Riemann equations, which is geometrically the condition that $\phi$ is constant in the $\bar{M}$ direction, i.e. is a holomorphic function on $M$.

The analogous splitting for $Q$ of dimension 4 is for 2-forms $F=F_{+}+F_{-}$, where $* F_{+}=F_{+}$and $* F_{-}=-F_{-}, *$ being the Hodge $*$-operator and depending only on the conformal structure (see [19] for more details on holomorphic conformal structures and *-operators). This decomposition corresponds dually in the tangent bundle to two families of totally null 2-planes, 
called by Penrose [25] $\alpha$-planes and $\beta$-planes, each family being parametrized by a Riemann sphere. A 2-form is self-dual $(* F=F)$ if and only if it vanishes on every $\beta$-plane. For a general holomorphic conformal structure there is an integrability condition [26] (the vanishing of part of the Weyl curvature) which ensures that these infinitesimal $\beta$-planes fit together to produce global $\beta$ surfaces. In this case the 3-dimensional parameter space is called the (dual) curved twistor space [26]. The quadric $Q$ is conformally flat and so has a dual twistor space $\mathbb{P}^{*}$ (and a twistor space parametrizing $\alpha$-planes), although the identification of this space is rather easier in this case [25]. The self-dual sourceless Maxwell equations are

$$
d F=0 \text { and } * F=F .
$$

These are a clear analogue of the Cauchy-Riemann equations for $n=2$, and the point of the Ward correspondence [31] is that the geometric interpretation provided by complexification carries over to Maxwell's equations and, by the same arguments, to self-dual Yang-Mills equations. More precisely, there is locally always a potential $\Phi$, i.e. a 1 -form such that $d \Phi=F$, and this is unique up to gauge freedom $\Phi \mapsto \Phi+d g$ for any function $g$. This is better interpreted as a line bundle with connection $\nabla$, where $\Phi$ is the connection 1-form and $F$ is the curvature. To say that $F$ is self-dual is to say that $\nabla$ is flat on $\beta$-surfaces. Thus, the line-bundle pushes down to a line-bundle on $\mathbb{P}^{*}$. This is one direction of the Ward correspondence.

For $S^{4}$ there is the alternative approach of Atiyah [1] which uses the Newlander-Nirenberg theorem to produce the holomorphic bundle on twistor space. The argument via complexification, however, is rigorous since the ellipticity of the equations assures real-analyticity (cf. Wells [32]).

Another example of complexification as used in twistor theory is Newman's $\mathscr{H}$-space [23] and the closely related hypersurface twistors of Penrose [25, 28]. In this context it is interesting to note that certain calculations in the smooth category can be avoided by means of complexification. To be more precise, Penrose observes that, associated to any space-like hypersurface in space-time, there is a CR-structure on the space of null geodesics meeting the hypersurface. Moreover, in the case of real-analytic space-time and hypersurface, it is possible to realize the space of null geodesics as a hypersurface in a 3-dimensional complex manifold, whence the CR-structure. It follows that the natural geometric definition of the CR-structure given by Penrose for the smooth case is automatically integrable since this is a calculation $[3,20]$ whose outcome is clearly independent of the regularity of the data. Conversely, LeBrun [20] has used this example to exhibit CR-manifolds not realizable as embedded hypersurfaces. Other examples from twistor theory are due to Hill, Penrose, and Sparling [29]. These are based on the general principle, due to Sparling, that holomorphic results of twistor theory can always be replaced by corresponding CR-statements in the case of smooth data.

3. Harmonic maps. If $\phi: M \rightarrow N$ is a smooth mapping between oriented Riemannian manifolds then the energy of $\phi$ is defined as

$$
E(\phi)=\int_{M} \frac{1}{2} \operatorname{trace} d \phi \wedge * d \phi,
$$


where $*: \Omega^{1}\left(\phi^{*} T N\right) \rightarrow \Omega^{m-1}\left(\phi^{*} T N\right)$ is the Hodge $*$-operator and the trace is taken with respect to the metric on $N$. Variation of $\phi$ gives as Euler-Lagrange equations for this functional

$$
\nabla(* d \phi)=0,
$$

where $\nabla: \Omega^{m-1}\left(\phi^{*} T N\right) \rightarrow \Omega^{m}\left(\phi^{*} T N\right)$ is the pull-back of the Levi-Civita connection on $N$. Such a critical mapping is called harmonic (see Eells and Lemaire [10] for a comprehensive review article). $\nabla(* d \phi)$ is the Hodge dual of the more usual tension. In local coordinates on $N$, maintaining a more abstract notation on $M$,

$$
\nabla(* d \phi)=d * d \phi^{i}+\Gamma_{j k}^{i} d \phi^{j} \wedge * d \phi^{k}
$$

where $\Gamma_{j k}^{i}$ are the Christoffel symbols of $N$.

There is a clear analogy with the Yang-Mills action and equations (an analogy familiar to many mathematicians and physicists (the latter being interested in harmonic maps under the name of " $\mathbb{C P}_{n}$-model", " $\sigma$-model", or "current algebra")). For harmonic maps, $d \phi$ is the analogue of the gauge field $F$ (the curvature of a vector bundle connection $\nabla$ ) and $\nabla(* d \phi)=0$ replaces $\nabla(* F)=0$. For gauge theories there is the Bianchi identity $\nabla F=0$. The corresponding identity holds in the harmonic case because $\nabla(d \phi)=\nabla\left(\phi^{*} \delta\right)$ $=\phi^{*} \nabla \delta$, where $\delta \in \Omega^{1}(T N)$ on $N$ is the tautological section (Kronecker delta) and $\nabla \delta$ is exactly the torsion of $\nabla$. For Yang-Mills, dimension 4 is special in that the equations are conformally invariant and, moreover, there are, by virtue of the Bianchi identity, special solutions comprising self-dual $F$, i.e. $F=* F$. As explained in \$2, twistor theory, via complexification, gives a natural geometric way of describing such solutions. It is natural to ask whether there is a corresponding theory for harmonic maps (cf. the use of complex analysis by Weierstrass in his construction of minimal surfaces in $\mathbb{R}^{3}$ (see Hitchin [15])).

The special dimension for harmonic maps is $\operatorname{dim} M=2$ for then the *-operator is conformally invariant and $M$ need therefore only be a Riemann surface. If $M$ were Lorentzian then $*^{2}=1$, and one can investigate the geometric significance of the harmonic equations by breaking $d \phi$ into self-dual and anti-self-dual parts. For Riemann $M$, however, $*^{2}=-1$ and, just as in Gauss' proof of the existence of isothermal parameters, a similar geometric interpretation is only possible after complexification. To obtain special solutions parallel to Yang-Mills, $N$ must first be complex (or almost complex) so that $* d \phi=i d \phi$ makes sense and, moreover, $\nabla$ should be complex linear, i.e. $N$ should be Kähler. In this case if $\phi$ is holomorphic, i.e. $* d \phi=i d \phi$, then

$$
\nabla(* d \phi)=\nabla(i d \phi)=i \nabla(d \phi)=0,
$$

so $\phi$ is harmonic. If $d \phi$ is split into self-dual and anti-self dual parts,

$$
d \phi=\partial \phi+\bar{\partial} \phi
$$

then $\phi$ is harmonic if and only if $\nabla(\partial \phi)=0$, or, equivalently, $\nabla(\bar{\partial} \phi)=0$.

This complexifies as follows. A holomorphic mapping $\Phi: \mathbb{C} M \rightarrow \mathbb{C} N$ is said to be harmonic if and only if $\nabla(* d \phi)=0$. More generally, $\mathbb{C} N$ can be replaced by an arbitrary complex manifold with torsion-free holomorphic 
connection, i.e. the reality structure does not enter into the definition of harmonic. Since $\nabla(d \Phi)=0, \Phi$ is harmonic $\Leftrightarrow \nabla(\partial \Phi)=0 \Leftrightarrow \nabla(\bar{\partial} \Phi)=0$. There is possible confusion here because $\partial \Phi$ in this context is not the complexification of $\partial \phi$ for $\phi: M \rightarrow N$, since, in particular, the complex structure on $N$ has been ignored. In order to reconcile this confusion in case $N$ has a complex structure, write $\Phi=(f, g): \mathbb{C} M \rightarrow N \times \bar{N}=\mathbb{C} N$. Then $\Phi$ is real, i.e. is the complexification of $\phi: M \rightarrow N$, if and only if $f(z, \bar{w})=\overline{g(w, \bar{z})}$ and $\partial \phi$ then complexifies to $\partial f$ or, equivalently, $\bar{\partial} g$, whereas $\partial \Phi$ is the pair $(\partial f, \partial g)$. However, as observed in $\S 3$, the Kähler condition on $N$ is reflected on C $N$ exactly as the condition that $\nabla$ preserve the splitting $\Theta=\Theta^{1,0} \oplus \Theta^{0,1}$. Thus, $\Phi$ is harmonic if and only if

$$
(\nabla(\partial f)=0 \text { or } \nabla(\bar{\partial} f)=0) \text { and }(\nabla(\partial g)=0 \text { or } \nabla(\bar{\partial} g)) \text {. }
$$

One of these four equivalent conditions is that $\nabla(\partial f)=0=\nabla(\bar{\partial} g)$, the complexification of $\nabla(\partial \phi)=0$.

Note that complexification is justified in the case of mappings between real-analytic Riemannian manifolds since, by ellipticity of the field equations (see Eells and Lemaire [10]), a harmonic map is automatically real-analytic. In particular, this is a bona fide procedure for the case of harmonic mappings from a Riemann surface into complex projective space.

Temporarily ignoring any real structure, a harmonic mapping to the complexification (for $M$ a Riemann surface) has a good geometric interpretation. Let $\Omega^{p, q}$ denote the bundle of holomorphic forms on $\mathbb{C} M$ of degree $p$ on $M$ and $q$ on $\bar{M}$. Then

$$
\nabla: \Omega^{1,0}\left(\Phi^{*} T \subset N\right) \rightarrow \Omega^{1,1}\left(\Phi^{*} T \subset N\right)
$$

may be considered as a connection in the $\bar{M}$-direction since it satisfies the Leibnitz rule $\nabla(f s)=f \nabla s+\bar{\partial} f \otimes s$. Because $\bar{M}$ is 1 -dimensional, $\nabla$ is flat and so trivializes $\Omega^{1,0}\left(\Phi^{*} T C N\right)$ in the $\bar{M}$-direction. Thus, $\Omega^{1,0}\left(\phi^{*} T C N\right)$ pushes down to a bundle on $M$ and the harmonic equation $\nabla(\partial \Phi)=0$ now says that $\partial \Phi \in \Omega^{1,0}\left(\Phi^{*} T C N\right)$ pushes down to a section of this bundle on $M$. In particular, this proves:

Proposition. Suppose $M$ is a Riemann surface and $\Phi: \mathbb{C} M \rightarrow \mathbb{C} N$ is harmonic. Then the zero-set of $\partial \Phi$ consists of the fibres of $\mu: \mathbb{C} M \rightarrow M$ over a discrete set of points (unless $\partial \Phi \equiv 0$ ) and thus, by rescaling, $\partial \Phi$ defines a complex direction at $\Phi(x)$ even if $\partial \Phi(x)=0$.

If $N$ is Kähler then a stronger version of this proposition is available. In this case write $\Phi=(f, g)$ and recall that $\Phi$ harmonic implies, in particular, that $\nabla(\partial f)=0$. Thus, by exactly the same argument, $\partial f$ can be rescaled (unless $\partial f \equiv 0$ ) to define a complex direction in $N$. Similar conclusions apply to $\bar{\partial} f, \partial g$, and $\bar{\partial} g$. The following theorem follows by taking $\Phi$ to be real.

THEOREM. Suppose $\phi: M \rightarrow N$ is a harmonic map from a Riemann surface to a Kähler manifold. Then $\partial \phi$ (resp. $\bar{\partial} \phi)$ is either identically zero or has only isolated zeros of finite order and so defines a complex direction by rescaling. 
Relative connections, i.e. defined along the fibres of some mapping (in this case $\mu: \mathbb{C} M \rightarrow M$ ), are familiar from twistor theory (e.g. Manin [21]). Indeed, in this lower dimension, $M$ can be considered as its own (dual) "twistor space", i.e. $M$ parametrizes the anti-self-dual curves in $C M$.

Another aspect of this geometric interpretation of harmonic maps is their propagation from "null data" (cf. Penrose [27]): a harmonic map near $x \in \mathbb{C} M$ is determined by its restriction to the null cone through $x$, and this restriction can be arbitrary. To be more precise, suppose $(p, q) \in M \times \bar{M}$ and $f$ and $g$ are holomorphic functions with values in $\mathbb{C} N$ such that $f(p)=g(q)$. Then there is a unique harmonic $\Phi: U \rightarrow \mathbb{C} N$ for some neighbourhood $U$ of $(p, q)$ such that $\Phi(z, q)=f(z)$ and $\Phi(p, w)=g(w)$. This is clear from $\nabla(\partial \Phi)=0$ if one thinks of propagating $\Phi$ away from $\{z=p\}$ using parallel transport from $\{w=q\}$ to determine the derivative $\partial \Phi$ in the $z$-direction. If, for example, $N=R$ so $\mathbb{C} N=\mathbb{C}$, then this becomes the statement that, locally, every harmonic $\mathbb{C}$-valued function on $M$ is the sum of a holomorphic function and an antiholomorphic function. This special case is a low-dimensional version of the Kirchoff integral formula $[25,27]$ for the propagation of massless fields on space-time.

The remainder of this article concerns the construction of harmonic maps from Riemann surfaces into complex projective space due to Burns [4], Din and Zakrzewski [7, 8], and Eells and Wood [11, 12]. They also show that if the domain is the Riemann sphere then this construction constitutes a classification. This additional analytic argument is not aided by complexification and will be omitted here. As far as the construction goes there are two ingredients. The first is an analytic one, namely the theorem proved earlier in this section. The second ingredient is just algebraic manipulation of the field equations specific to the range being complex projective space. This manipulation is not aided by complexification.

Let $\langle$,$\rangle be an Hermitian form on a complex vector space V$. Let $H$ denote the Hopf bundle on $\mathbb{P}(V)$ and $T$ the tangent bundle. Then there is the Euler sequence

$$
\begin{array}{lll}
\mathbb{1} & \hookrightarrow & V \otimes H \rightarrow T \\
\Psi & & \Psi \\
f & \mapsto & f Z,
\end{array}
$$

where 1 is the trivial complex line bundle and $Z$ denotes the canonical section of $V \otimes H$ given by the inclusion $H^{*} \subset V$. The Hermitian form provides a splitting

$$
V \otimes H \ni P \mapsto\langle P, Z\rangle /\|Z\|^{2} \in \mathbb{1}
$$

and hence an Hermitian metric, the Fubini-Study metric, on the tangent space as a subspace $\{\langle P, Z\rangle=0\} \subset V \otimes H$. The corresponding metric connection is given by

$$
\nabla P=d P-[\langle d P, Z\rangle Z+\langle d Z, Z\rangle P] /\|Z\|^{2}
$$


where the derivatives are taken nonprojectively, noting that the resulting form is appropriately homogeneous: if $Z \mapsto \lambda Z$ then $P \mapsto \lambda P$ and $\nabla P \mapsto \lambda \nabla P$. Also note that $\langle\nabla P, Z\rangle=0$ as required. Now suppose $\phi: M \rightarrow \mathbb{P}(V)$ for some Riemann surface $M$. Using the same notation for $\phi^{*} T$ one can write

$$
\partial \phi=\partial R-\langle\partial R, R\rangle R /\|R\|^{2},
$$

where $R: M \rightarrow V$ is any lifting of $\phi$. Note that the right-hand side as a section of $T^{1,0} M \otimes V \otimes \phi^{*} H$ is independent of choice of $R$ : if $R \mapsto \lambda R$ then

$$
\begin{aligned}
\partial \phi & \mapsto \partial(\lambda R)-\langle\partial(\lambda R), \lambda R\rangle \lambda R /\|\lambda R\|^{2} \\
& =\lambda \partial R-\lambda\langle\partial R, R\rangle R /\|R\|^{2}+(\partial \lambda) R-(\partial \lambda) R \\
& =\lambda \partial \phi .
\end{aligned}
$$

Since the following calculations are local on $M$ it is convenient to regard $\partial$ and $\bar{\partial}$ as coordinate derivates rather than anything more invariant. In particular, they commute, rather than anticommute, and higher derivatives such as $\partial^{2}$ make good sense. These local statements and constructions, however, are easily seen to be really coordinate free (and hence extend to global constructions). By substituting the above expression for $\partial \phi$ into the formula for $\nabla$ it follows that $\phi$ is harmonic if and only if

$$
\partial \bar{\partial} R-[\langle\partial R, R\rangle \bar{\partial} R+\langle\bar{\partial} R, R\rangle \partial R] /\|R\|^{2}=\mu R
$$

for some function $\mu$.

For $R: M \rightarrow V$ define

$$
D R=\partial R-\langle\partial R, R\rangle R /\|R\|^{2} \text { and } \bar{D} R=\bar{\partial} R-\langle\bar{\partial} R, R\rangle R /\|R\|^{2},
$$

noting that $D(\lambda R)=\lambda D R$ and $\bar{D}(\lambda R)=\lambda \bar{D} R$. If $S: M \rightarrow V$ also, then write $R \perp S$ to mean $\langle R, S\rangle=0$.

LEMMA. If $R \perp S$ then $\langle D R, S\rangle+\langle R, \bar{D} S\rangle=0$.

ProOF.

$$
\begin{gathered}
\left\langle\partial R-\langle\partial R, R\rangle R /\|R\|^{2}, S\right\rangle+\left\langle R, \bar{\partial} S-\langle\bar{\partial} S, S\rangle S /\|S\|^{2}\right\rangle \\
=\langle\partial R, S\rangle+\langle R, \bar{\partial} S\rangle=\partial\langle R, S\rangle=0 .
\end{gathered}
$$

It is easy to verify that if $R$ is a lift of a harmonic $\phi$ then

$$
D \bar{D} R=\alpha R \text { and } \bar{D} D R=\beta R
$$

for some functions $\alpha$ and $\beta$. Conversely, if these equations hold and, in addition, $D R$ and $\bar{D} R$ are linearly independent or one of them vanishes, then it is not difficult to check that $\phi$ is harmonic. In particular, this is the case if $\phi$ is isotropic, i.e.

$$
D^{p} R \perp \bar{D}^{q} R \text { for all } p, q \geqslant 1 \text {. }
$$

Using the above lemma one can verify that this is equivalent to saying that $\operatorname{span}\left\{R, \partial R, \partial^{2} R, \ldots\right\}$ meets $\operatorname{span}\left\{R, \bar{\partial} R, \bar{\partial}^{2} R, \ldots\right\}$ orthogonally to $\operatorname{span}\{R\}$. Indeed, isotropy implies that $\ldots, \bar{D}^{2} R, \bar{D} R, R, D R, D^{2} R, \ldots$ are all mutually 
orthogonal. For example, $D R \perp R$ by construction so, from the lemma, $\left\langle D^{2} R, R\right\rangle=-\langle D R, \bar{D} R\rangle=0$.

Theorem (Burns, Din, ZAKRZEWSKi, Eells, Wood). If $\phi$ is harmonic and isotropic then so is $D \phi$.

PROoF. The statement of the theorem first needs some explanation. First represent $\phi$ by a lift $R: M \rightarrow V$ and compute $D R$. It may be that $D R \equiv 0$, in which case $\partial \phi \equiv 0$, i.e. $\phi$ is antiholomorphic. If this is not the case then the previous theorem asserts that $D R$ may be rescaled so as to map into $V-\{0\}$. The corresponding map into $\mathbb{P}(V)$ is called $D \phi$.

To check isotropy of $D \phi$ means to check that

$$
\ldots, \bar{D}^{2}(D R), \bar{D}(D R), D R, D(D R), D^{2}(D R), \ldots
$$

are mutually orthogonal. Since $\phi$ is harmonic, $\bar{D} D R=\beta R$ for some function $\beta$ so this becomes

$$
\ldots, \beta \bar{D} R, \beta R, D R, D^{2} R D^{3} R, \ldots,
$$

which are mutually orthogonal by isotropy of $\phi$. To check harmonicity,

$$
D \bar{D}(D R)=D(\beta R)=\beta D R,
$$

which is one of the required equations. For the other equation, first note that, by commuting the first two derivatives and using the one just proved, it follows that

$$
\bar{D} D(D R) \in \operatorname{span}\left(R, D R, D^{2} R\right) .
$$

Now use the lemma:

$$
\left\langle R, \bar{D} D^{2} R\right\rangle=-\left\langle D R, D^{2} R\right\rangle=0
$$

and $\bar{D} D^{2} R \perp D^{2} R$ by construction.

As a particular case of a harmonic isotropic $\phi$ to use in this theorem, one can start with $\phi$ holomorphic. Then $D \phi, D^{2} \phi, D^{3} \phi, \ldots$ will provide a family of nonholomorphic harmonic maps terminating in an antiholomorphic map. By isotropy this will happen in at most $n$ steps for $n$ the dimension of $\mathbb{P}(V)$. It will happen in less than $n$ steps only if $\phi(M)$ is contained in a lower-dimensional projective space. The operator $\bar{D}$ can be used to progress in the other direction along this family.

In twistor theory there is a description of the full Yang-Mills equations (rather than just self-dual) due to Green, Isenberg and Yasskin [16], and Witten [33]. An analogy of this description for harmonic maps appears to be lacking.

\section{REFERENCES}

1. M. F. Atiyah, Geometry of Yang-Mills fields, Lezioni Fermiane, Scuola Normale Superiore Pisa, Pisa, 1979.

2. F. Bruhat and H. Witney, Quelques propriétés fondamentales des ensembles analytiques réels, Comment. Math. Helv. 33 (1959), 132-160.

3. R. Bryant, Space-time and CR-manifolds, Trans. Amer. Math. Soc. (to appear).

4. D. Burns, Harmonic maps from $\mathbb{C P}^{1}$ to $\mathbb{C P}{ }^{n}$, Harmonic Maps (Proc. Conf., Tulane Univ.), Lecture Notes in Math., vol. 949, Springer-Verlag, 1982, pp. 48-56.

5. S.-S. Chern, An elementary proof of the existence of isothermal parameters on a surface, Proc. Amer. Math. Soc. 6 (1955), 771-782. 
6. D. M. DeTurck and J. L. Kazdan, Some regularity theorems in Riemannian geometry, Ann. Sci. École Norm. Sup. (4) 14 (1981), 249-260.

7. A. M. Din and W. J. Zakrzewski, Properties of the general $\mathbb{C P}^{n-1}$ model, Phys. Lett. B 95 (1980), 419-422.

8. , General classical solutions in the $\mathbb{C P}^{n-1}$ model, Nuclear Phys. B 174 (1980), 397-406.

9. B. Eckmann and A. Frölicher, Sur l'intégrabilité des structures presque complexes, C.R. Acad. Sci. Paris 232 (1951), 2284-2286.

10. J. Eells and L. Lemaire, A report on harmonic maps, Bull. London Math. Soc. 10 (1978), $1-68$.

11. J. Eells and J. C. Wood, The existence and construction of certain harmonic maps, Symposia Mathematica XXVI (Rome, 1980), Academic Press, New York and London, 1982, pp. 123-138.

12. , Harmonic maps from surfaces to complex projective spaces, Warwick Univ., 1981 (preprint).

13. H. Grauert, On Levi's problem and the imbedding of real analytic manifolds, Ann. of Math. (2) 68 (1958), 460-472.

14. S. W. Hawking, The path-integral approach to quantum gravity, General Relativity: An Einstein Centenary Survey, Cambridge Univ. Press, 1979, pp. 746-789.

15. N. J. Hitchin, Monopoles and geodesics, Comm. Math. Phys. 83 (1982), 579-602.

16. J. Isenberg, P. B. Yasskin and P. S. Green, Non-self-dual gauge fields, Phys. Lett. B 78 (1978), 462-464.

17. N. Koiso, Einstein metrics and complex structures, Invent. Math. 73 (1983), 71-106.

18. C. R. LeBrun, $\mathscr{H}$-space with a cosmological constant, Proc. Roy. Soc. London. Ser. A. 380 (1982), 171-185.

19. Spaces of complex null geodesics in complex-Riemannian geometry, Trans. Amer. Math. Soc. 278 (1983), 209-231.

20. Twistor CR manifolds and three-dimensional conformal geometry, Trans. Amer. Math. Soc. 284 (1984), 601-616.

21. Yu. I. Manin, Gauge fields and holomorphic geometry, Current Problems in Mathematics, vol. 17, Akad. Nauk SSSR, Vsesayuz. Inst. Nauchn. i Tekhn. Informatsii Moscow, 1981, pp. 3-55.

22. A. Newlander and L. Nirenberg, Complex analytic coordinates in almost complex manifolds, Ann. of Math. (2) 65 (1957), 391-404.

23. E. T. Newman, Heaven and its properties, Gen. Relativity Gravitation 7 (1976), 107-111.

24. L. Nirenberg, Lectures on linear partial differential equations, CBMS Regional Conf. Ser. in Math., No. 17, Amer. Math. Soc., Providence, R. I., 1973.

25. R. Penrose, Twistor theory, its aims and achievements, Quantum Gravity: An Oxford Symposium, Clarendon Press, Oxford, 1975, pp. 268-407.

26. Non-linear gravitons and curved twistor theory, Gen. Relativity Gravitation 7 (1976), $31-52$.

27. _ Null hypersurface initial data for classical fields of arbitrary spin and for general relativity, Gen. Relativity Gravitation 12 (1980), 225-264.

28. R. Penrose and R. S. Ward, Twistors for flat and curved space-time, General Relativity and Gravitation (A. Held, ed.), Vol. II, Plenum Press, New York and London, 1980, pp. 283-328.

29. R. Penrose, Physical space-time and non-realizable CR-structures, Bull. Amer. Math. Soc. (N.S.) 8 (1983), 427-448.

30. M. Sato, T. Kawai and M. Kashiwara, Microfunctions and pseudodifferential equations, Lecture Notes in Math., vol. 287, Springer-Verlag, 1973, pp. 265-529.

31. R. S. Ward, On self-dual gauge fields, Phys. Lett. A 61 (1977), 81-82.

32. R. O. Wells, Jr., The conformally invariant Laplacian and the instanton vanishing theorem, Seminar on Differential Geometry (S.-T. Yau, ed.), Ann. Math. Stud. No. 102, Princeton Univ. Press, Princeton, N. J., 1982, pp. 483-498.

33. E. Witten, An interpretation of classical Yang-Mills theory, Phys. Lett. B 77 (1978), 394-398.

Mathematical Institute, 24 - 29 St. Giles', OXford OX1 3LB, England 